\title{
Resultados preliminares do implante de lente intra-ocular fácica Artisan para correção de miopia
}

\author{
Preliminary results of Artisan phakic intraocular lens implantation tocorrect myopia
}

\author{
Leonardo Akaishi ${ }^{1}$ \\ Herval Cavalcanti Martins ${ }^{2}$ \\ Rodrigo Rezende ${ }^{3}$ \\ Patrick Frensel de Moraes Tzelikis ${ }^{4}$
}

Trabalho realizado no Serviço de Córnea, Catarata e Doenças Externas do Hospital Oftalmológico de Brasília HOB - Brasília (DF) - Brasil.

${ }^{1}$ Mestre em Oftalmologia, Chefe do Serviço de Córnea e Catarata do Hospital Oftalmológico de Brasília - HOB - Brasília (DF) - Brasil.

${ }^{2}$ Residente em Oftalmologia do HOB - Brasília (DF) Brasil.

Residente em Oftalmologia do HOB - Brasília (DF) Brasil.

${ }^{4}$ Médico Assistente do Serviço de Córnea e Catarata do HOB - Brasília (DF) - Brasil.

Endereço para correspondência: Patrick F. Tzelikis SQN 203 - Bl. G - Apto. 405 - Asa Norte - Brasília (DF) CEP 70833-070

Recebido para publicação em 16.11.2005

Última versão recebida em 28.11.2006

Aprovação em 06.12.2006

Nota Editorial: Depois de concluída a análise do artigo sob sigilo editorial e com a anuência dos Drs. Marcelo Carvalho da Cunha e Belquiz Rodrigues A. Nassaralla sobre a divulgação de seus nomes como revisores, agradecemos sua participação neste processo.

\begin{tabular}{|c|}
\hline RESUMO \\
\hline 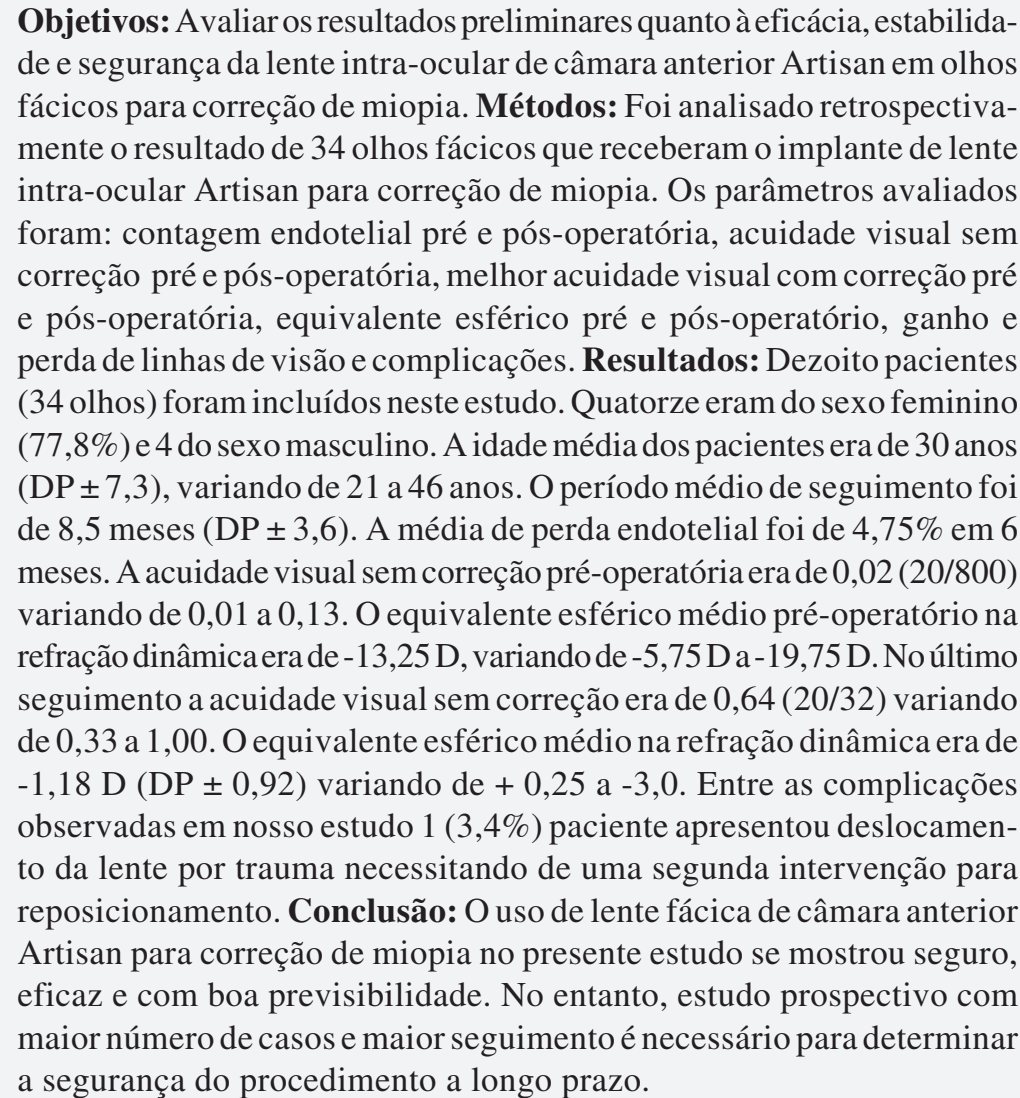 \\
\hline
\end{tabular}

Descritores: Implante de lente intra-ocular; Lentes intra-oculares; Miopia/cirurgia

\section{INTRODUÇ̃̃̃O}

A rápida recuperação visual, o baixo índice de complicações e a alta previsibilidade para correção de diferentes graus de ametropia têm feito do LASIK (laser assisted in situ keratomileusis) a técnica mais utilizada para tratamento da miopia ${ }^{(1)}$. No entanto, apesar de todo o entusiasmo inicial, o LASIK apresenta complicações principalmente quando utilizado para correção de altas ametropias. Complicações como ectasia iatrogênica, aberrações ópticas, intenso "glare" noturno, complicações relacionadas ao "flap", e perda de melhor acuidade visual corrigida já foram descritas ${ }^{(2-3)}$.

Em virtude das limitações das opções disponíveis para correção de altas ametropias, novas alternativas como o implante de lente intra-ocular fácica 
vem sendo pesquisado em vários centros. A idéia de se utilizar lentes fácicas de câmara anterior foi primeiramente apresentada na década de 50 com Strampellii ${ }^{(4)}$, sendo recentemente reintroduzida por Fechner ${ }^{(5)}$. Nos últimos anos se observa um crescente interesse no uso de lentes intra-oculares fácicas devido a sua boa previsibilidade, estabilidade e por não comprometer a acomodação ${ }^{(6)}$.

$\mathrm{O}$ uso de lente intra-ocular de câmara anterior de fixação iriana foi inicialmente desenhado por Worst para correção cirúrgica da afacia ${ }^{(7)}$. Em 1986, Fechner et al, criaram uma lente para correção de miopia, baseada na lente de Worst ${ }^{(5)}$. Desde então as lentes foram modificadas e aperfeiçoadas com o intuito de reduzir as complicações e melhorar os resultados visuais. $\mathrm{O}$ presente estudo apresenta os resultados iniciais obtidos com o uso de lente intra-ocular de câmara anterior Artisan (Ophtec BV, Groninen, Holanda) em pacientes com miopia.

\section{MÉTODOS}

Foram avaliados retrospectivamente os dados de todos os pacientes portadores de miopia submetidos à cirurgia de implante de lente intra-ocular de câmara anterior Artisan para correção de miopia no Serviço de Córnea, Catarata e Refrativa do Hospital Oftalmológico de Brasília (HOB).

Os dados coletados incluem sexo, idade, profundidade de câmara anterior (CA), contagem endotelial (CE) pré e pós-operatória, acuidade visual sem correção (AVSC) pré e pós-operatória, melhor acuidade visual com correção (AVCC) pré e pósoperatória, equivalente esférico (EE) pré e pós-operatório, astigmatismo pré e pós-operatório e complicações. Neste estudo, as medidas de acuidade visual foram registradas em decimal (ex: $20 / 50=0,4$ ), sendo que os pacientes com visão pior que 20/800 formam registrados como apresentando acuidade visual de 0,01 (ex: conta dedos ou movimento de mãos $=0,01$ ). Microscopia especular foi realizada na maioria dos pacientes sendo utilizando o aparelho de microscopia especular de não-contato (NonCon Robo, Konan, Tóquio, Japão). Os critérios de inclusão para o implante da lente Artisan em nosso trabalho era idade superior a 18 anos, profundidade de câmara anterior maior que $2,6 \mathrm{~mm}$ e contagem de célula endotelial maior que 2.000 céls $/ \mathrm{mm}^{2}$. Todos os pacientes com história de cirurgia ocular prévia, catarata, glaucoma, retinopatia diabética, olho único e doenças sistêmicas graves foram excluídos do estudo.

A lente utilizada no presente estudo foi a lente Artisan (Ophtec BV, Groninen, Holanda) que vem sendo usada desde 1986 em olhos fácicos ${ }^{(8)}$. É uma lente de peça única, constituída de polimetilmetacrilato (PMMA), com uma parte central (óptica) que possui um desenho convexo-côncavo com diâmetro de 5,00 ou $6,00 \mathrm{~mm}$, e uma região háptica apresentando uma pequena falha na qual se realiza o encravamento na meia-periferia da íris. O diâmetro total da lente é de $8,50 \mathrm{~mm}$, com o poder dióptrico variando de $-3,00$ a $-23,50 \mathrm{D}$ (dioptrias) dependendo da parte óptica utilizada.

Todas as lentes - Artisan - foram implantadas pelo mesmo cirurgião (L.A.). No pré-operatório os pacientes utilizam colírio antibiótico de quinolona de $4^{\underline{a}}$ geração (Vigamox ${ }^{\circledR}$ Alcon ou Zymar $^{\circledR}$ Allergan), 4 vezes por dia (6/6 h), 2 dias antes da cirurgia. No dia da cirurgia era utilizado pilocarpina $2 \%$ para provocar miose pupilar aproximadamente 30 minutos antes da cirurgia, 1 gota de $10 \mathrm{em} 10$ minutos por 3 vezes. A anestesia em todos os casos era tópica, com instilação de cloridrato de lidocaína geléia a 2\% (Astra Zeneca, Argentina) sem vaso-constritor no fundo de saco conjuntival, 30 minutos antes da cirurgia por 2 vezes em um intervalo de 10 minutos. Por via endovenosa era utilizado 1,0 mg de midazolam (Roche, Brasil) $5,0 \mathrm{mg} / \mathrm{ml}$. Após assepsia e anti-sepsia da pele com iodo-povidona a $10 \%$ (Cinord Sul, Brasil), equivalente a $1 \%$ de iodo ativo, um campo plástico adesivo era colocado em contato com as pálpebras dos pacientes, de forma a isolar os cílios do campo operatório, e o blefarostato posicionado. Uma solução de iodo-povidona a 3\% (Ophthalmos, São Paulo) foi usada com finalidade anti-séptica no fundo de saco conjuntival após anestesia tópica. Uma incisão límbica tunelizada era feita superiormente com 5,25 e 6,25 $\mathrm{mm}$ de extensão de acordo com diâmetro da lente a ser implantada. Injetava-se viscoelástico (Viscoat ${ }^{\circledR}$, Alcon, USA), a seguir a lente era inserida na câmara anterior e rodada horizontalmente sendo centralizada sobre a pupila. Com ajuda de uma pinça, fixava-se a parte óptica da lente e realizava-se o encravamento de parte da íris ao háptico da lente. A posição correta da lente no centro da pupila era verificada cuidadosamente. Ao final do procedimento realizava-se uma iridectomia profilática às 12 horas e o fechamento da incisão com nylon 10-0. No período pósoperatório, o paciente era orientado a utilizar topicamente: diclofenaco de sódio, (Acular ${ }^{\circledR}$, Allergan Produtos Farmacêuticos, São Paulo, Brasil) quatro vezes ao dia por quatro semanas, moxifloxacino $\left(\right.$ Vigamox $\left.^{\circledR}\right)$ ou gatifloxacino $\left(\right.$ Zymar $\left.^{\circledR}\right) 4$ vezes ao dia por 10 dias, e acetato de prednisolona 0,1\% (Predfort $^{\circledR}$, Alcon Laboratórios do Brasil, São Paulo, Brasil) 4 vezes ao dia por 4 semanas. Medicação analgésica via oral também era prescrita em caso de dor. Em todos os pacientes o objetivo era alcançar a emetropia.

Toda análise estatística foi realizada através do programa SPSSX (SPSS Inc, Chicago, IL).

\section{RESULTADOS}

Dezoito pacientes (34 olhos) foram submetidos ao implante da lente intra-ocular de câmara anterior Artisan e analisados retrospectivamente. Quatorze eram do sexo feminino $(77,8 \%)$ e 4 do sexo masculino (22,2\%). A idade média dos pacientes era de 30 anos, variando de 21 a 46 anos (desvio-padrão $\mathrm{DP} \pm 7,3$ ). Dezesseis pacientes $(88 \%)$ foram operados bilateralmente. Foram realizados $16(47,1 \%)$ implantes no olho direito (OD) e $18(52,9 \%)$ no olho esquerdo (OS). Os pacientes foram seguidos por um período médio de 8,5 meses ( $\mathrm{DP} \pm 3,6$ ), variando de 3 a 15 meses.

A média de profundidade de CA pré-operatória era de $3,39 \mathrm{~mm}$ (DP $\pm 0,27 \mathrm{~mm}$ ), variando de 2,9 a $4,0 \mathrm{~mm}$. A CE média préoperatória era de 2.587 céls $/ \mathrm{mm}^{2}\left(\mathrm{DP} \pm 237,6\right.$ céls $\left./ \mathrm{mm}^{2}\right)$, varian- 
do de 2.093 a 3.072 céls $/ \mathrm{mm}^{2}$. Ao final de 6 meses a CE média era de $2.464,6$ céls $/ \mathrm{mm}^{2}\left(\mathrm{DP} \pm 217,3\right.$ céls $\left./ \mathrm{mm}^{2}\right)$, variando de 2.023 a 2.910 céls $/ \mathrm{mm}^{2}$. A média de perda endotelial em 6 meses foi de $4,75 \%$. Não se observou diferença estatística entre as medidas pré e pós-operatórias $(\mathrm{p}>0,05)$ utilizando-se o teste $\mathrm{t}$ para diferença entre as duas médias.

No exame pré-operatório observava-se uma média de AVSC de 0,03 (20/600) ( $\mathrm{DP} \pm 0,03)$, variando de 0,01 a 0,13. A média da melhor AVCC pré-operatória era de 0,72 (20/28) $(\mathrm{DP} \pm 0,22)$, variando de 0,25 a 1,00 . O EE médio pré-operatório na refração dinâmica era de $-13,20 \mathrm{D}(\mathrm{DP} \pm 3,39)$, variando de $-5,75 \mathrm{D}$ a $-19,75 \mathrm{D}$.

No seguimento de 1 mês pós-operatório $(\mathrm{n}=34)$, a média da AVSC era de 0,57 (20/35) (DP $\pm 0,18)$, variando de 0,20 a 1,00. A média da melhor AVCC era de $0,89(20 / 22)(\mathrm{DP} \pm 0,13)$, variando de 0,50 a 1,00 e o EE médio pós-operatório na refração dinâmica era de $-1,30 \mathrm{D}(\mathrm{DP} \pm 0,80)$, variando de $+0,50 \mathrm{D}$ a $-3,50 \mathrm{D}$. No seguimento de 6 meses de pós-operatório $(\mathrm{n}=28)$, a média da AVSC era de 0,64 (20/31) (DP $\pm 0,22)$, variando de 0,33 a 1,00. A média da melhor AVCC era de $0,91(20 / 22)(\mathrm{DP} \pm 0,14)$, variando de 0,50 a 1,00. O EE médio pós-operatório na refração dinâmica era de $-1,18 \mathrm{D}(\mathrm{DP} \pm 0,92)$, variando de $+0,25 \mathrm{D}$ a $-3,00 \mathrm{D}$.

No último seguimento, a média da AVSC era de 0,59 (20/33) (DP $\pm 0,23)$, variando de 0,20 a 1,00 . A média da melhor AVCC era de $0,90(20 / 22)(\mathrm{DP} \pm 0,12)$ e o EE médio era de $-1,30 \mathrm{D}(\mathrm{DP} \pm 0,94)$. As diferenças observadas entre as médias pré e pós-operatórias da AVSC, AVCC e EE foram significativas em todos os períodos estudados ( 1 mês, 6 meses e final) utilizando-se um nível de significância de 1\% (Tabela 1).

No pré-operatório observava-se um astigmatismo médio de $-1,80 \pm 1,33 \mathrm{D}$ (variando de plano a $-5,25 \mathrm{D}$ ). Após a cirurgia a

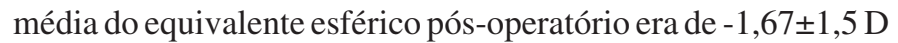
(variando de plano a $-5,50 \mathrm{D}$ ) no primeiro mês, $-1,49 \pm 1,25 \mathrm{D}$ (variando de plano a $-5,00 \mathrm{D}$ ) no sexto mês, e $-1,53 \pm 1,10 \mathrm{D}$ (variando de plano a 5,0 D) último seguimento. Não foi encontrado nenhuma diferença estatisticamente significativa do astigmatismo pós-operatório nos meses 1, 6 e último seguimento, baseado no teste t Student $(\mathrm{p}>0,05)$.

No presente trabalho, após o seguimento médio de 8,5 meses, 11 olhos $(39,3 \%)$ apresentavam $\pm 0,50 \mathrm{D}$ de emetropia e 16 olhos $(57,1 \%) \pm 1,00 \mathrm{D}$. Ao exame final $67,7 \%$ dos casos $(23$ olhos) apresentavam AVSC de 0,5 (20/40) ou melhor e $14,7 \%$ (5 olhos) AVSC de 1,00 (20/20). A melhor AVCC era de 0,50 (20/ 40) ou melhor em $96,5 \%$ dos olhos e 1,00 (20/20) em $67,9 \%$ dos olhos. A análise da acuidade visual através da perda ou ganho de linhas de visão é um parâmetro que pode ser utilizado para se avaliar a segurança da cirurgia. Em nosso trabalho quando comparamos a AVCC pré-operatória com a pós-operatória observa-se que nenhum olho perdeu linhas de visão, 14 olhos $(41,2 \%)$ mantiveram a mesma acuidade visual, 15 olhos $(44,1 \%)$ ganharam 1 linha de visão, 4 olhos (11,8\%) ganharam 2 linhas de visão e 1 olho (2,9\%) ganhou 3 linhas de visão.

Entre as complicações observadas em nosso estudo, 1 olho $(3,4 \%)$ desenvolveu deslocamento da lente intra-ocular após trauma ocular contuso por agressão no oitavo mês pósoperatório de cirurgia sendo necessário a realização de uma segunda intervenção cirúrgica para reposicionamento da lente. Ao exame de microscopia especular o paciente apresentava uma contagem endotelial de 2.486 céls $/ \mathrm{mm}^{2}$ previamente a cirurgia de implante da lente Artisan, tendo sido reduzida para 2.313 céls $/ \mathrm{mm}^{2}$ no terceiro mês e após a cirurgia de reposicionamento da lente passou a apresentar uma contagem endotelial de 1.890 céls $/ \mathrm{mm}^{2}$. O mesmo paciente apresentou um novo deslocamento de lente intra-ocular ( 2 meses após a reoperação), segundo o paciente após coçar o olho intensamente, sendo realizado novo reposicionamento. Ao final do último seguimento o paciente apresentava uma contagem endotelial de 1.470 céls $/ \mathrm{mm}^{2}$ e córnea transparente. Nenhum paciente desenvolveu glaucoma agudo por bloqueio pupilar ou glaucoma por dispersão pigmentar, no entanto, a presença de pigmento sobre a lente implantada foi observada em 3 casos $(8,8 \%)$.

Apesar de todos os pacientes estarem satisfeitos com o resultado visual final, a presença de fenômenos de disfotopsia como halos e "glare" foram relatados por 4 pacientes (22\%), sendo que em apenas $1(5,5 \%)$ existia uma dificuldade em dirigir no período noturno.

\section{DISCUSSÃO}

Vários trabalhos têm mostrado excelentes resultados com o uso de lentes fácicas. Entre as vantagens do uso destas lentes encontra-se a preservação da acomodação, a reversibilidade da cirurgia sendo que a lente pode ser removida a qualquer momento, a estabilidade do procedimento visto que não ocorre alteração na estrutura da córnea, e a relativa simplicidade no implante da lente ${ }^{(8-9)}$. Em comparação aos procedimentos refrativos corneanos, não existe preocupação quanto ao processo de cicatrização, regressão ou complicações com o "flap". No entanto, complicações como síndrome de dispersão pigmentar, glaucoma, catarata, e descompensação corneana por comprometimento endotelial podem ocorrer ${ }^{(8-10)}$.

\begin{tabular}{|c|c|c|c|c|}
\hline Média & Pré-operatório & 1 mês $(n=34)$ & 6 meses $(n=28)$ & Final 8,5 meses \\
\hline EE & $-13,20 \mathrm{D}$ & $-1,30 \mathrm{D}$ & $-1,18 \mathrm{D}$ & $-1,30 \mathrm{D}$ \\
\hline AVSC & $0,03(20 / 600)$ & $0,57(20 / 35)$ & $0,64(20 / 31)$ & $0,59(20 / 33)$ \\
\hline AVCC & $0,72(20 / 28)$ & $0,89(20 / 22)$ & $0,91(20 / 22)$ & $0,90(20 / 22)$ \\
\hline CE & 2.587 céls $/ \mathrm{mm}^{2}$ & - & 2.464 céls $/ \mathrm{mm}^{2}$ & - \\
\hline
\end{tabular}


No presente estudo, o uso de lentes fácicas de câmara anterior com fixação iriana teve sucesso na redução da miopia. É possível observar uma redução no EE médio pré-operatório dinâmico de -13,20 D (DP $\pm 3,39)$ para -1,18 D (DP $\pm 0,92)$, ao final de um seguimento de 6 meses. Resultados semelhantes foram relatados na literatura em um trabalho realizado na Espanha em $2004^{(9)}$, nos quais os autores apresentaram uma redução no EE médio dinâmico pré-operatório de -16,17 D ( $\mathrm{DP} \pm 2,75)$ para $-0,85 \mathrm{D}(\mathrm{DP} \pm 1,37)$ em 137 olhos operados após 6 meses de cirurgia.

Quando observamos a proporção de linhas ganhas ou perdidas, é possível verificar que $58,8 \%$ dos olhos submetidos ao implante da lente Artisan obtiveram um ganho de pelo menos 1 linha de visão na acuidade visual corrigida final, este ganho está provavelmente relacionado ao aumento da imagem ao nível da retina. Nenhum paciente perdeu linha de visão. Em contraste, o LASIK quando comparado a este procedimento, apresenta uma perda esperada de 2 ou mais linhas de visão entre $3 \%$ a $5 \%$ dos olhos operados com alta miopia (miopia $>6,00 \mathrm{D}$ ) ao final de 6 meses $^{(11)}$. Resultados semelhantes foram relatados em 2002 no estudo multicêntrico americano do implante de $\operatorname{Artisan}^{(10)}$, com $58 \%$ dos olhos ganhando 1 a 2 linhas de visão ao final de 6 meses de acompanhamento.

Quanto à contagem endotelial foi possível observar em nosso trabalho uma perda média de $4,75 \%$ no número de células endoteliais ao final de 6 meses. Diversos estudos vêm apresentando uma perda endotelial média semelhante, variando entre $3,30 \%$ a $10,6 \%{ }^{(9,12-13)}$. Trata-se de um resultado bastante favorável, uma vez que é aceitável e esperado uma perda de células endoteliais após facoemulsificação entre 7\% a $10 \%$ nos primeiros 6 meses $^{(14)}$.

Foi observada apenas uma grande complicação no presente estudo: deslocamento da lente intra-ocular pós-trauma. No caso aqui apresentado uma das regiões de encravamento da lente na íris solta após o trauma ocular contuso, sendo necessário uma nova intervenção cirúrgica para reposicionamento da lente por duas vezes. Em ambos os procedimentos de encravamento da lente na íris, uma boa parte da mesma foi utilizada o que não evitou o seu desprendimento por duas ocasiões. Não foi encontrado nenhum caso de glaucoma por bloqueio pupilar ou glaucoma por dispersão pigmentar, apenas depósito de pigmento na lente provavelmente relacionado ao ato cirúrgico, visto que os mesmos não progrediram. Fenômenos de disfotopsia foram relatados por $22 \%$ dos pacientes no pós-operatório sendo na sua grande maioria leve a moderada, com um único paciente referindo dificuldade em dirigir a noite. Mesmo assim, todos os pacientes estavam satisfeitos com o resultado visual final.

Em conclusão, o uso de lentes fácicas de câmara anterior com fixação iriana - Artisan - para correção de miopia no presente estudo se mostrou segura, fácil e com boa previsibilidade. No entanto, um estudo prospectivo com maior número de casos e maior seguimento é necessário para determinar a segurança do procedimento a longo prazo.

\section{ABSTRACT}

Purpose: To observe the preliminary results based on the efficacy, stability and safety of Artisan anterior chamber intraocular lens for the correction of myopia in the first 6 months. Methods: A retrospective study of 18 patients (34 eyes) that received the implantation of Artisan anterior chamber intraocular lens for the correction of myopia was conducted. Best visual preoperative acuity, best visual postoperative acuity, dynamic preoperative and postoperative refraction, loss and improvement of lines of vision and complications were evaluated. Results: Eighteen patients (34 eyes) were included in this study. Fourteen patients were females $(77.8 \%)$ and 4 were males. The mean age was 30 years $(\mathrm{SD} \pm 7.3)$, range from 21 to 46 years. They were followed up for an average of 8.5 months $(\mathrm{SD} \pm 3.6)$. The mean endothelial cell loss was $4.75 \%$ at 6 months follow-up. The average preoperative uncorrected visual acuity was 0.02 (20/800) range from 0.01 to 0.13 . The average preoperative spherical equivalent was $-13.25 \mathrm{D}$, range from $-5.75 \mathrm{D}$ to $-19,75 \mathrm{D}$. On the last follow-up, uncorrected visual acuity was 0.64 (20/32), range from 0.33 to 1.00 ; the average spherical equivalent was $-1.18 \mathrm{D}(\mathrm{DP} \pm 0.92)$ range from +0.25 to -3.0 . Complications were observed in 1 case $(3.4 \%)$ of our study, in one patient who had a dislocation of the lens after an ocular trauma. Conclusion: The use of Artisan anterior chamber intraocular lens in phakic eyes for the correction of myopia was safe, effective and predictable. However, a larger prospective study with a higher number of cases and longer follow-up is necessary to determine long-term safety of the lens.

Keywords: Lens implantation, intraocular; Lenses, intraocular; Myopia/surgery

\section{REFERÊNCIAS}

1. Pallikaris IG, Siganos DS. Excimer laser in situ keratomileusis and photorefractive keratectomy for the correction of high myopia. J Refract Corneal Surg. 1994;10(5):498-510.

2. Seiler T, Koufala K, Richter G. Iatrogenic keratectasia after laser in situ keratomileusis. J Refract Surg. 1998;14(3):312-7.

3. Stulting RD, Carr JD, Thompson KP, Waring GO 3rd, Wiley WM, Walker JG. Complications of laser in situ keratomileusis for the correction of myopia. Ophthalmology. 1999;106(1):13-20.

4. Strampelli B. Sopportabilitá di lenti acriliche in camera anteriore nella afachia e nei vizi de refrazione. Ann Ottamol Clin Ocul. 1954;80(2):75-82.

5. Fechner PU, van der Heijde GL, Worst JG. [Intraocular lens for the correction of myopia of the phakic eye]. Klin Monatsbl Augenheilkd. 1988;193(1):29-34. German.

6. Assetto V, Benedetti S, Pesando P. Collamer intraocular contact lens to correct high myopia. J Cataract Refract Surg. 1996;22(5):551-6.

7. Worst JG. Iris Claw Lens. J Am Intraocul Implant Soc. 1980;6(2):166-7.

8. Fechner PU, Die Irisklauen-Linse, Klin Monatsbl Augenheilkd. 1987;191(1):26-9.

9. Menezo JL, Peris-Martinez C, Cisneros AL, Martinez-Costa R. Phakic intraocular lenses to correted high myopia: Adatomed, Staar, and Artisan. J Cataract Refract Surg. 2004;30(1):33-44.

10. Maloney RK, Nguyen LH, John ME. Artisan phakic intraocular lens for myopia: short-term results of a prospective, multicenter study. Ophthalmology. 2002;109(9):1631-41.

11. Knorz MC, Wiesinger B, Liermann A, Seiberth V, Liesenhoff H. Laser in situ 
keratomileusis for moderate and high myopia and myopic astigmatism. Ophthalmology. 1998;105(5):932-40.

12. De Souza RF, Forseto A, Nose R, Belfort R Jr, Nose W. Anterior chamber intraocular lens for high myopia: five year results. J Cataract Refract Surg. $2001 ; 27(8): 1248-53$.
13. Perez-Santonja JJ, Bueno JL, Zato MA. Surgical correction of high myopia in phakic eyes with Worst-Fechner myopia intraocular lenses. J Refract Surg. 1997;13(3):268-81. Discussion 281-4.

14. Bourne WM, Nelson LR, Hodge DO. Central corneal endothelial cell changes over a ten-year period. Invest Ophthalmol Vis Sci. 1997;38(3):779-82.

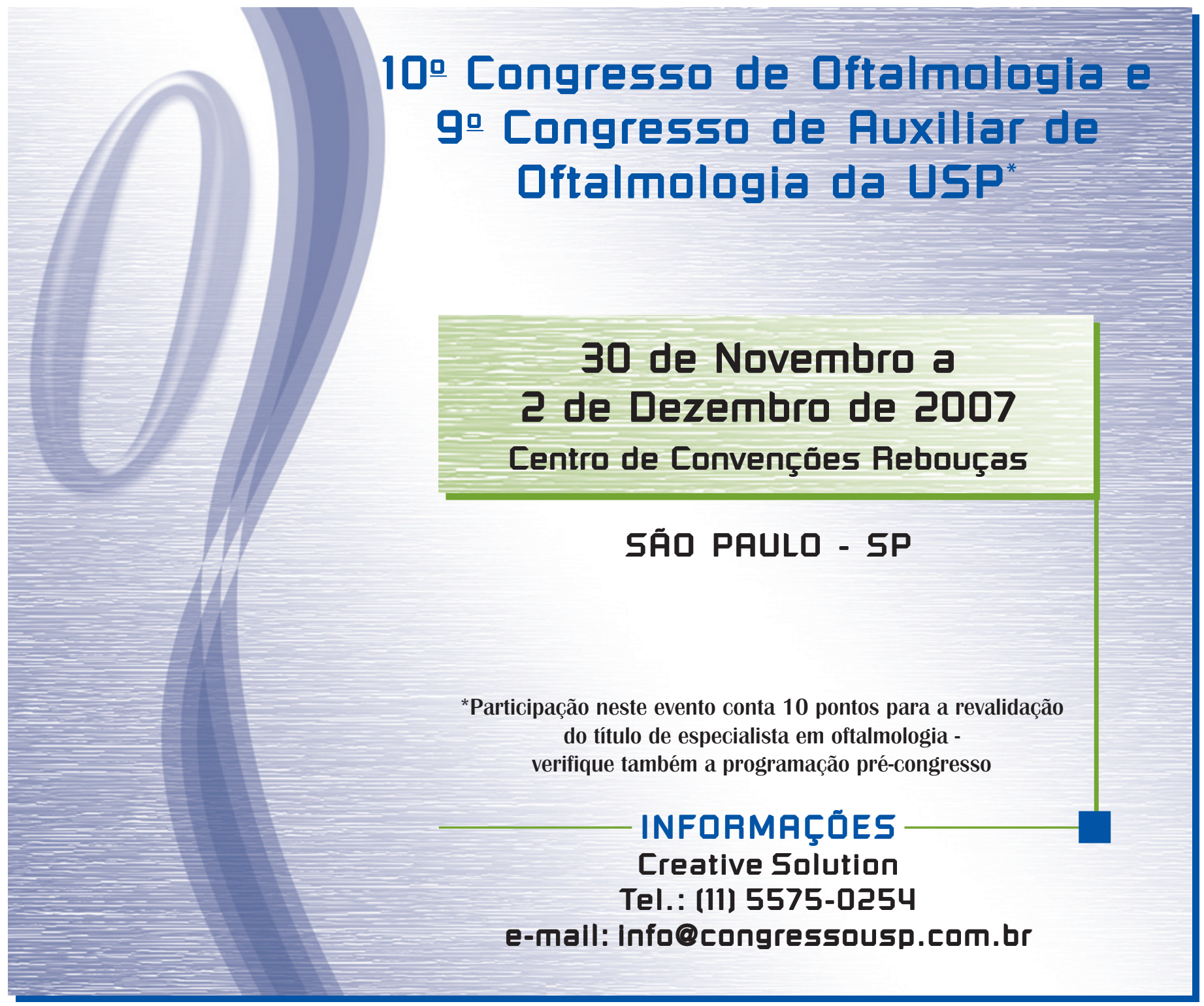

